\title{
EVALUACIÓN DE LA REMOCIÓN DE ARSÉNICO EN AGUA SUPERFICIAL UTILIZANDO FILTROS DOMICILIARIOS
}

\begin{abstract}
Guillermo F. Villa-Gonzales ${ }^{1, a}$, Carlos Huamaní-Pacsii, ${ }^{1, b}$, Manuel Chávez-Ruiz ${ }^{1, c}$, José A. Huamaní-Azorza $^{1, d}$
RESUMEN

El objetivo del estudio fue evaluar filtros domiciliarios para remover arsénico presente en agua proveniente del río Sama. El sistema estuvo compuesto de un filtro tradicional de arena y un filtro con el material adsorbente de hierro cerovalente estabilizado en quitosano. La evaluación del sistema fue por un período de 12 semanas en 18 puntos de uso; las primeras ocho semanas se determinó la concentración de arsénico del agua filtrada empleando tiras reactivas, y las cuatro últimas fueron a través de espectrometría de absorción atómica (EAA-FIAS). Durante las primeras ocho semanas se redujo la concentración de arsénico en el rango de 0,005 y 0,025 mg/L, y durante las cuatro últimas en el rango de 0,001 y $0,052 \mathrm{mg} / \mathrm{L}$, a partir de una concentración promedio de 0,51 mg/L. Se demostró que el sistema de filtración es eficiente para remover arsénico a condiciones naturales.
\end{abstract}

Palabras clave: Arsénico; Población Rural; Ingestión de Líquidos; Perú (fuente: DeCS BIREME).

\section{EVALUATION OF THE ARSENIC REMOVAL IN SUPERFICIAL WATERS USING HOME FILTERS}

\begin{abstract}
The objective of the study was to evaluate home filters to remove arsenic present in waters coming from the Sama river. The system consisted of a traditional sand filter and a filter with the adsorbent zerovalent iron material stabilized in quitosan. The evaluation of the system lasted for a period of 12 weeks in 18 points of usage; the first eight weeks the arsenic concentration of the filtered water was determined using reactive strips, and the last four assessment was made through atomic absorption spectrometry (AAS). During the first eight weeks the arsenic concentration was reduced in a range of 0.005 and $0.025 \mathrm{mg} / \mathrm{L}$, and during the last four weeks in the range of 0.001 and $0.052 \mathrm{mg} / \mathrm{L}$, from an average concentration of $0.51 \mathrm{mg} / \mathrm{L}$. This research showed that the filtration system is efficient to remove arsenic to natural conditions
\end{abstract}

Keywords: Arsenic; Rural Population; Drinking Water (source: MeSH NLM).

\section{INTRODUCCIÓN}

En algunos países de Latinoamérica como Argentina, Chile y Perú más de cuatro millones de personas beben a diario agua con arsénico (As) en concentraciones superiores a $0,01 \mathrm{mg} / \mathrm{L}$, lo cual es un serio problema de salud pública por sus consecuencias en la salud ${ }^{(1,2)}$. Según la Organización Panamericana de la Salud, en el Perú por lo menos unas 250000 personas beben agua con niveles de arsénico por encima del valor permitido en el Decreto Supremo $N^{\circ} 031$ 2010-SA, es decir, mayor a $0,01 \mathrm{mg} / \mathrm{L}^{(3,4)}$.

El informe sobre Calidad del agua para consumo, reporta que dentro de la jurisdicción de la Dirección Regional de
Salud de Tacna (DIRESA Tacna), existen un total de 312 comunidades, de las cuales 30 son urbanas y 282 son rurales. De estas comunidades, el Programa de Vigilancia de la Calidad del Agua para consumo humano (PVICA), reportó que 126 de 221 comunidades cuentan con servicio de abastecimiento de agua, por lo cual los habitantes de 92 comunidades consumen agua directamente de la fuente, es decir, superficial o subterránea ${ }^{(5)}$.

El consumo prolongado de agua con arsénico produce la enfermedad conocida como hidroarsenismo crónico regional endémico (HACRE), esta provoca alteraciones cardíacas, vasculares y neurológicas, trastornos hepáticos, renales e hiperqueratosis cutánea, y en el peor de los casos, puede producir neoplasias o cáncer ${ }^{(6)}$.

\footnotetext{
Instituto Nacional de Salud. Lima, Perú.

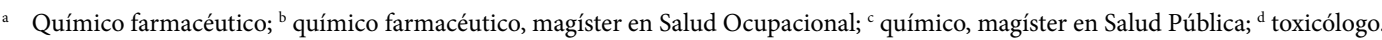
Parte de los resultados fueron presentados a manera de resumen en el X Congreso Científico Internacional del Instituto Nacional de Salud «Allin Kawsay» - Una Salud - One Health.

Recibido: 06/06/2018 Aprobado: 28/11/2018 En línea: 21/12/2018
}

Citar como: Villa-Gonzales GF, Huamaní-Pacsi C, Chávez-Ruiz M, Huamaní-Azorza JA. Evaluación de la remoción de arsénico en agua superficial utilizando filtros domiciliarios. Rev Peru Med Exp Salud Publica. 2018;35(4):652-6. doi:10.17843/rpmesp.2018.354.3715. 
En Argentina y Chile se han probado diversos métodos para remover el arsénico del agua destinada para el consumo humano, pero muchos de éstos funcionan en plantas de tratamiento y en algunos casos no se atienden aquellas personas que carecen de un abastecimiento directo o que viven a ciertas distancias una de otra, además son métodos costosos y poco sostenibles en el tiempo ${ }^{(7-9)}$. Frente a esta dificultad una alternativa eficiente es la remoción de metales pesados mediante el proceso de adsorción.

Se ha descrito que la síntesis de hierro cerovalente encapsulado en nanoesferas de quitosano es un método de bajo costo para la absorción de iones arsénico, con óptimos resultados a $\mathrm{pH}$ ácido y básico, y que no interfiere con otros iones ${ }^{(10)}$. El uso actual de las nanopartículas de hierro cerovalente sobre matrices poliméricas es una de las técnicas más prometedoras en la remoción de contaminantes. Por lo descrito, el objetivo fue diseñar y evaluar filtros domiciliarios para la remoción de arsénico utilizando hierro cerovalente estabilizado en quitosano acoplado a un filtro tradicional de arena, en una localidad rural peruana.

\section{EL ESTUDIO}

El estudio fue realizado en la localidad de Coruca, localizado en el distrito de Inclán (provincia y departamento de Tacna), con latitud sur 17 $38^{\prime} 17^{\prime \prime}$ y longitud oeste 70²7'34", conformado por tres anexos: Yarascay, Coruca y Sambalay (Figura 1). Según la información brindada por el responsable del establecimiento de salud local, en 2016,

\section{MENSAJES CLAVE}

Motivación para realizar el estudio. La población rural de Tacna que no tiene acceso a agua potable ingiere agua contaminada naturalmente por arsénico, lo cual significa una exposición crónica y por consecuencia deterioro progresivo de la salud.

Principales hallazgos. El filtro domiciliario, compuesto por un filtro tradicional de arena y un filtro con el adsorbente a base de quitosano y hierro cerovalente, logra remover arsénico del agua, proveniente del rio Sama, hasta en un $99,8 \%$.

Implicancias. Es necesario realizar investigaciones dirigidas hacia la reducción de metales pesados en el agua destinada para consumo humano, pues su contaminación representa un riesgo latente.

Coruca tuvo una población de 114 habitantes, de los cuales 41 viven permanentemente, de estos, el $90 \%$ se dedica principalmente a la actividad ganadera y agrícola.

En el periodo junio-julio 2016, se seleccionó aleatoriamente 18 viviendas de manera proporcional al número de vivienda de los tres anexos: siete filtros en Yarascay, nueve filtros en Coruca, y dos filtros en Sambalay. En estos anexos se provee agua superficial procedente del río Sama.

Se solicitó los permisos al Gobierno Regional de Tacna en coordinación con la DIRESA Tacna, para la instalación de los sistemas de filtración en los tres anexos de Coruca. Los participantes fueron invitados a participar de manera
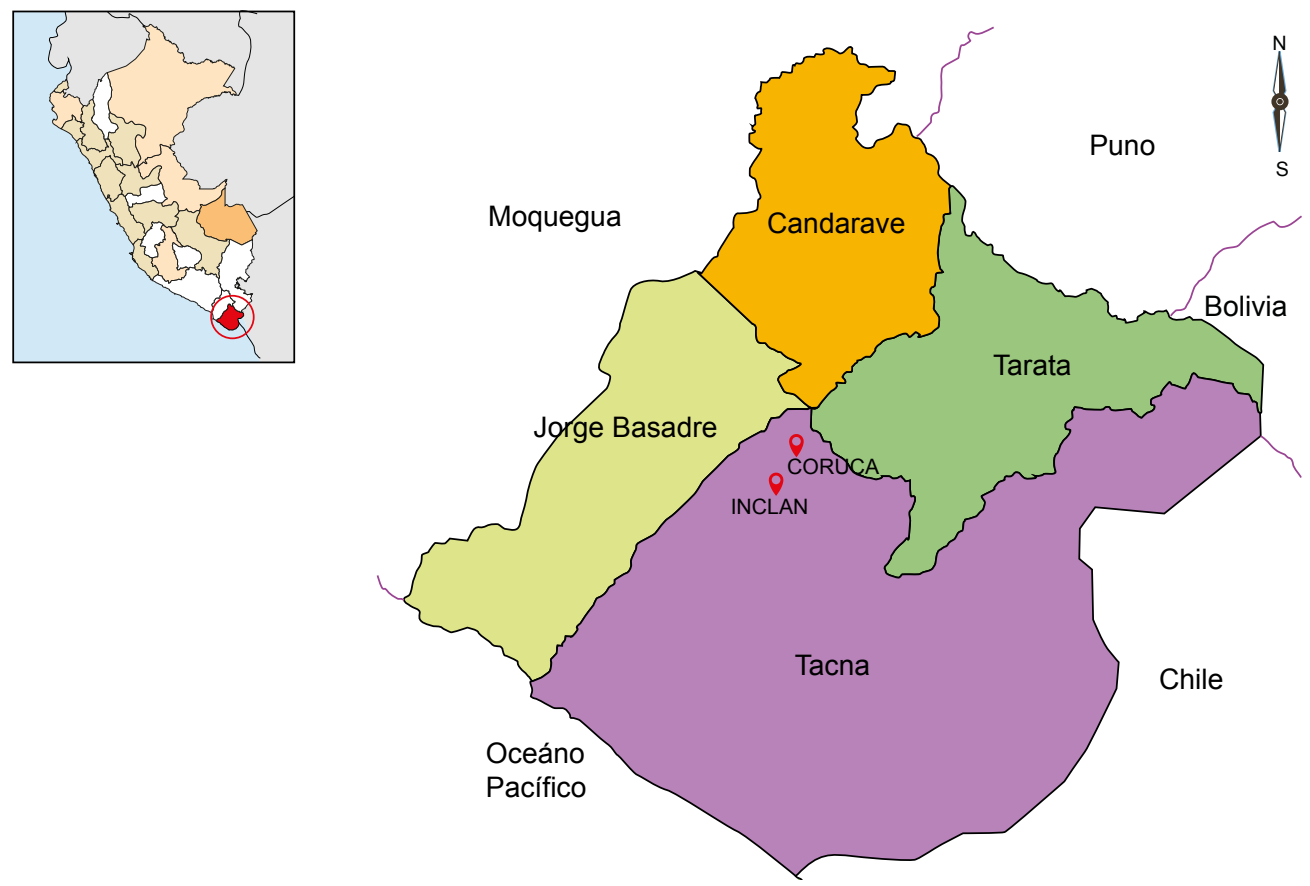

Figura 1. Mapa físico del departamento de Tacna y ubicación de la localidad de Coruca en distrito de Inclán. 
voluntaria. Los sistemas de filtración fueron instalados dentro de las viviendas, y la instalación estuvo a cargo de los investigadores y personal técnico de la DIRESA Tacna. Al término de la instalación se midió los flujos de salida del sistema para verificar su correcta operación, la Figura 2 muestra su instalación y un esquema del sistema de filtración.

La toma de muestra para la evaluación del sistema de filtración fue realizada por un técnico previamente capacitado por los investigadores. Las muestras colectadas del agua superficial y filtrada fueron analizadas mediante el procedimiento de toma y conservación de muestras del «Standard Methods» para exámenes de agua «APHAAWWA-WPCF-17 edition».

La concentración de arsénico total en las muestras de agua fue evaluado durante doce semanas, las primeras ocho semanas fue empleando el kit de determinación para arsénico semicuantitativo mediante tiras cuantitativas y las últimas cuatro mediante análisis de absorción atómica por el método basado en el Standard Methods con el equipo Espectrómetro de absorción atómica - Sistema de análisis de inyección de flujo (EAA-FIAS), marca Perkin Elmer, modelo AAnalyst 400 y FIAS 100 ejecutado con el programa WinLab 32.

La supervisión del óptimo funcionamiento de cada sistema de filtración se realizaba dos veces por semana y el encargado fue el técnico previamente entrenado. Los criterios para verificar el correcto funcionamiento fueron: flujos de salida y concentración de arsénico residual con las tiras semicuantitativas; la principal utilidad de esta prueba rápida fue de tener los datos in situ. La eficiencia fue calculada según la siguiente fórmula:

Eficiencia $=\frac{(\mathrm{Cc}-\mathrm{C} f) * 100}{\mathrm{Cc}}$

Donde: Cc: concentración control de arsénico en el agua superficial procedente del río Sama al inicio de las semanas $9,10,11$ y 12 según anexos; Cf: concentración final de arsénico.

El análisis estadístico incluyó la evaluación de la normalidad de datos con la prueba de Kolmogorov-Smirnov con corrección de Lilliefors considerando una significancia estadística como $p<0,05$. Asimismo, para la evaluación de la existencia o no de diferencia significativa entre la concentración de arsénico inicial y residual de los tres anexos de Coruca se empleó la prueba de $U$ de Mann Whitney.

\section{HALLAZGOS}

La concentración promedio de arsénico inicial fue de $0,51 \mathrm{mg} / \mathrm{L}$, y el arsénico residual, luego de pasar por los filtros, durante las primeras ocho semanas estuvo en un rango de 0,005 y $0,025 \mathrm{mg} / \mathrm{L}$. En las últimas cuatro semanas los valores de arsénico residual fluctuaron entre 0,001 y $0,052 \mathrm{mg} / \mathrm{L}$ (Tabla 1 ).
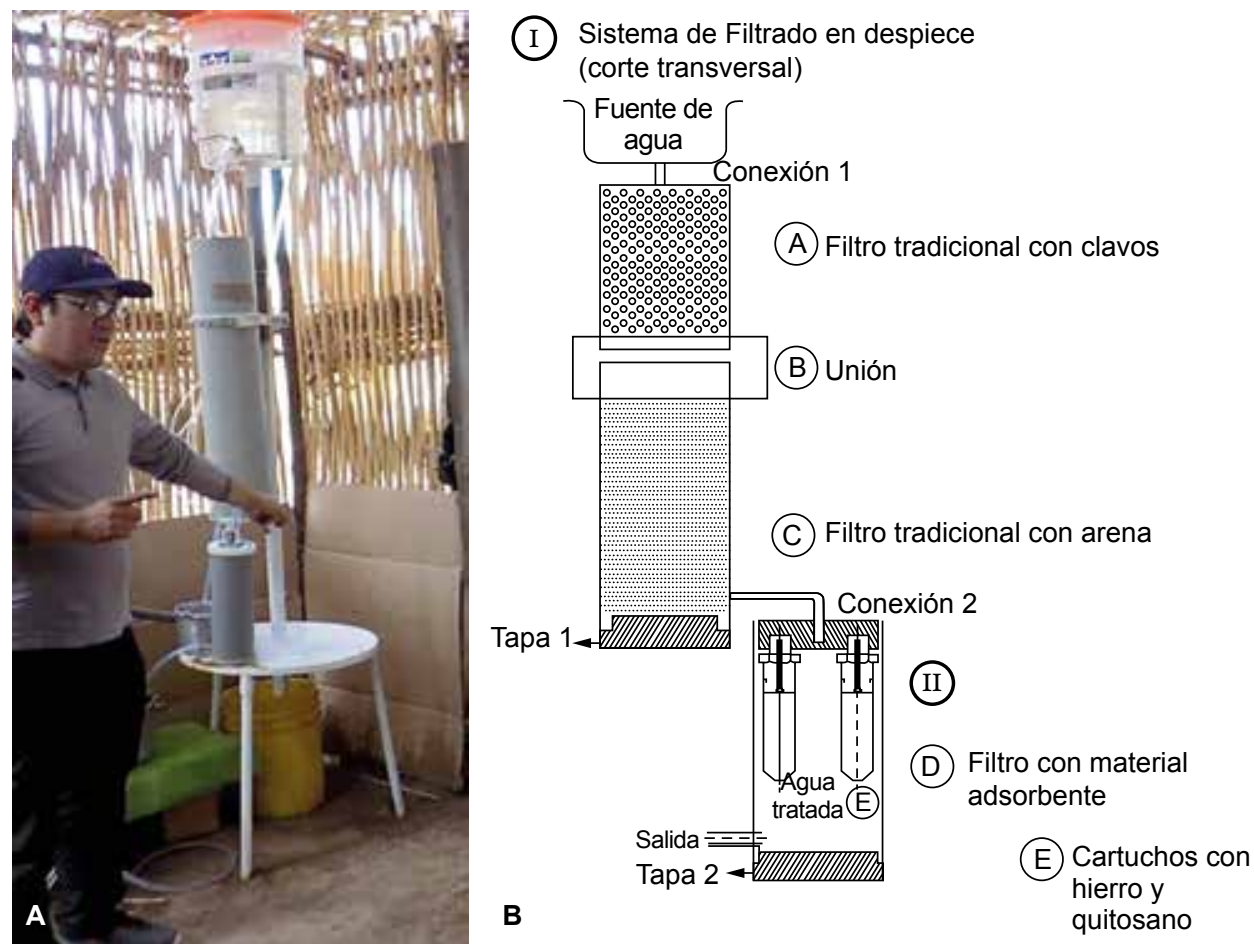

Figura 2. A. Instalación del filtro domiciliario; B. Esquema del sistema de filtración, compuesto por Filtro I, filtro tradicional de arena y virutas de hierro y Filtro II, material adsorbente de hierro cerovalente y quitosano. 
Tabla 1. Concentración de arsénico residual durante las cuatro últimas semanas del estudio.

\begin{tabular}{|c|c|c|c|c|}
\hline \multirow{2}{*}{ Característica } & \multicolumn{4}{|c|}{ Concentración de arsénico residual (mg/L) } \\
\hline & Semana 9 & Semana 10 & Semana 11 & Semana 12 \\
\hline Control (Cc) & 0,510 & 0,490 & 0,520 & 0,500 \\
\hline \multicolumn{5}{|l|}{ Código del filtro } \\
\hline 01-M & 0,020 & 0,011 & 0,006 & 0,046 \\
\hline 02-M & 0,022 & 0,015 & 0,018 & 0,032 \\
\hline 03-M & 0,005 & 0,002 & 0,001 & 0,010 \\
\hline 04-M & 0,005 & 0,002 & 0,005 & 0,008 \\
\hline 05-M & 0,037 & 0,029 & 0,038 & 0,031 \\
\hline 06-M & 0,043 & 0,013 & 0,014 & 0,021 \\
\hline 07-M & 0,052 & 0,037 & 0,030 & 0,042 \\
\hline 08-M & 0,029 & 0,023 & 0,014 & 0,026 \\
\hline 09-M & 0,042 & 0,024 & 0,045 & 0,050 \\
\hline $10-\mathrm{M}$ & 0,015 & 0,013 & 0,015 & 0,046 \\
\hline $11-\mathrm{M}$ & 0,015 & 0,010 & 0,031 & 0,044 \\
\hline $12-\mathrm{M}$ & 0,003 & 0,002 & 0,002 & 0,021 \\
\hline 13-M & 0,004 & 0,004 & * & 0,016 \\
\hline $14-\mathrm{M}$ & 0,050 & 0,026 & * & 0,031 \\
\hline $15-M$ & 0,035 & 0,023 & * & 0,010 \\
\hline $16-\mathrm{M}$ & 0,033 & 0,031 & * & 0,050 \\
\hline 17-M & 0,031 & 0,027 & * & 0,049 \\
\hline 18-M & 0,045 & 0,030 & 0,030 & 0,040 \\
\hline
\end{tabular}

* Menor al límite de detección del método de medición empleado.

Cc: concentración control de arsénico en el agua superficial procedente del río Sama al inicio de las semanas 9, 10,11 y 12 .

Los promedios de concentraciones de arsénico residual en las últimas cuatro semanas fueron $0,027 \mathrm{mg} / \mathrm{L}, 0,018 \mathrm{mg} / \mathrm{L}$, 0,014 mg/L, 0,032 mg/L, respectivamente (Figura 3), con un rango de eficiencia que va desde $89,8 \%$ hasta $99,8 \%$.

La Figura 3 muestra la distribución de los datos e indica que la menor concentración de arsénico en promedio se observa en la semana 11 con un valor de 0,014. Los filtros 03-M y

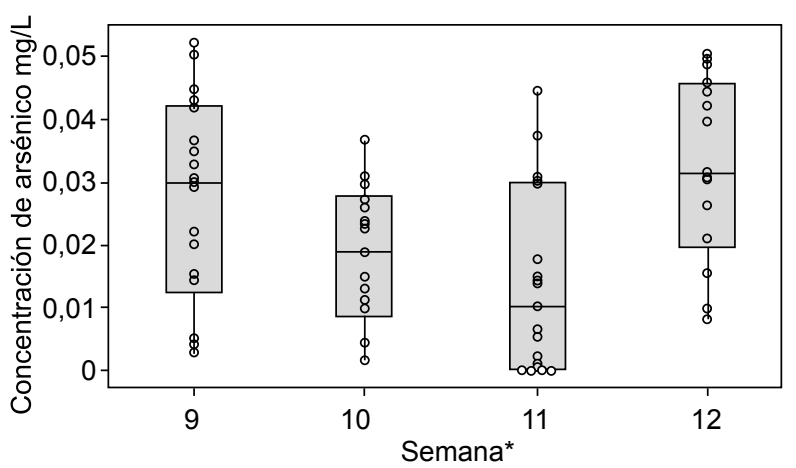

Figura 3. Concentraciones de arsénico residual durante las cuatro últimas semanas del estudio

* Media semana 9: 0,027 mg/L, media semana 10: 0,018 mg/L, media semana 11: 0,014 mg/L, media semana 12: 0,032 mg/L.
04-M logran remover arsénico con altas eficiencias, el filtro 03-M logró una eficiencia mínima de 98,0\% y una máxima de $99,8 \%$, por su parte, el filtro 04-M logró una eficiencia mínima de $98,3 \%$ y una máxima de $99,8 \%$. No obstante, el sistema que obtuvo menor eficiencia fue el filtro 07-M con $89,8 \%$ de remoción para la semana nueve.

\section{DISCUSIÓN}

Las eficiencias de los filtros 03-M y 04-M son concordantes con el estudio realizado en Vietnam del Norte, en el que se utilizó filtros de arena y hierro que lograron remover arsénico del agua desde una concentración inicial de 0,42 mg/L a $0,023 \mathrm{mg} / \mathrm{L}^{(11)}$, es decir una remoción del $94,5 \%$. Otro estudio en Vietnam también mostró que los filtros de arena podrían remover $80 \%$ hasta $95 \%$ de arsénico ${ }^{(12,13)}$, dichas eficiencias son a partir de agua subterránea a diferencia del presente estudio que utilizó agua superficial donde podría existir mayor presencia de sólidos totales e interferentes en la remoción de arsénico, tales como fosfatos, silicatos, entre otros.

Los filtros en estudio lograron remover arsénico del río Sama en un rango que va desde $0,001 \mathrm{mg} / \mathrm{L}$ hasta $0,052 \mathrm{mg} / \mathrm{L}$. El filtro de arena tradicional compuesta por arena, grava y clavos, prolonga la vida útil del segundo filtro el cual remueve arsénico de manera eficaz, debido a la presencia del 
adsorbente de hierro cerovalente estabilizado en quitosano. Su funcionamiento se basa en la liberación de hierro (II) a partir de hierro con valencia cero en presencia de oxígeno y este a su vez promueve la oxidación del arsénico (III) a (V), y a partir del hierro (II) se forma óxido de hierro (III) hidratado que adsorbe el arsénico del agua ${ }^{(14-16)}$.

Durante la ejecución del estudio se observó que el total de los 18 filtros durante las cuatro últimas semanas del estudio, muestran eficiencias variables, probablemente se deba al modo de operación de cada familia o a los volúmenes de filtración por día.

Los resultados de este estudio demuestran que los sistemas de filtración de agua superficial colocados en punto de uso son eficientes para la remoción de arsénico del agua, y que podrían tener gran aceptación por los pobladores de zonas rurales, que no cuentan con abastecimiento del recurso hídrico potabilizado, por su fácil funcionamiento y empleo sin gasto de energía eléctrica.

Se debe reconocer que el estudio estuvo limitado a la evaluación semicuantitativa del comportamiento del sistema de filtración durante las primeras ocho semanas y el análisis analítico instrumental de sólo las últimas cuatro semanas del estudio debido a las restricciones logísticas y económicas

Se recomienda ampliar el tiempo de evaluación para determinar la capacidad máxima de agua filtrada por cada unidad del sistema, regeneración de las mismas y número de veces que pueda ser reutilizado el adsorbente. También es necesario desarrollar este tipo de tecnologías bajo diferentes tipos de recurso hídrico, puesto que las condiciones hidrogeoquímicas varían de acuerdo a la ubicación geográfica.

Agradecimientos: Al equipo técnico y profesional de la Dirección Regional de Salud Tacna quienes brindaron las facilidades de planificación y ejecución del estudio a través del Programa de Vigilancia de Calidad del Agua, en especial al Dr. Claudio Ramírez, a la Blga. Juana Sosa, al Ing. Daniel Chire y al Ing. Julio Sotelo por su valioso apoyo y participación en el estudio.

Contribuciones de los autores: GFVG, CHP, MChR y JAHA han participado en la concepción del artículo, la recolección de datos, su redacción y aprobación de la versión final. GFVG realizó el análisis de datos, y obtuvo el financiamiento.

Fuentes de financiamiento: el proyecto fue financiado con fondos externos de la organización Grand Challenges de Canadá.

Conflictos de interés: los autores declaran no tener conflictos de interés.

\section{REFERENCIAS BIBLIOGRÁFICAS}

1. Mandal B, Suzuki K. Arsenic around the world: a review. Talanta. 2002;58(1):201-35.

2. Castro de Esparza M. Presencia de arsénico en el agua de bebida en América Latina y su efecto en la salud pública. In: International Congress: Natural Arsenic in Groundwaters of Latin America. México, México DC: 20-24 June 2006.

3. Castro de Esparza M. Arsénico en el agua de bebida de la población de los valles de Locumba y Chipe. Lima, Perú: OPS/CEPIS; 2002.

4. Sancha A, Castro de Esparza M. Arsenic status and handling in Latin America. Lima, Perú: Universidad de Chile, Grupo As de AIDIS/DIAGUA, CEPIS/OPS; 2000.

5. Ministerio de salud del Perú, Dirección Regional de Salud Tacna. Calidad del agua para consumo humano región Tacna 2011-2013. Tacna: Dirección Ejecutiva de Salud Ambiental, equipo de saneamiento básico, higiene alimentaria y zoonosis. Programa de Vigilancia de la Calidad del Agua para Consumo Humano; 2013.

6. Ministerio de Salud de la Nación Argentina, Programa Nacional de prevención y control de las intoxicaciones PRECOTOX. Módulo de capacitación para la atención primaria. Hidroarsenismo Crónico Regional Endémico (HACRE) [Internet]. Buenos Aires, Argentina: Ministerio de Salud de la Nación; 2011 [11 de enero de 2018]. Disponible en: http:// www.msal.gob.ar/images/stories/bes/
graficos/0000000332cnt-03-Capacit hidroarsenicismo.pdf

7. Instituto Mexicano de Tecnología del Agua. Remoción de arsénico de agua para consumo humano. Informe Final. México: IMTA; 1997.

8. Sancha M. Experiencia chilena en abatimiento de arsénico. Seminario Potabilización de Aguas con Arsénico y Flúor. $9^{\circ}$ Congreso Argentino de Saneamiento y Medio Ambiente. Córdoba-Argentina, junio, 1996.

9. Granada J, Cerda W, Godoy D, ESSAN El camino para reducir notoriamente el arsénico en el agua potable. Revista AIDIS 2003:44-9.

10. Gupta A, Yunus M, Sankararamakrishnan N. Zerovalent iron encapsulated chitosan nanospheres - A novel adsorbent for the removal of total inorganic Arsenic from aqueous systems. Chemosphere. 2012;86(2):1505. doi: 10.1016/j.chemosphere.2011.10.003.

11. Agusa T, Trang K, Lan M, Anh H, Tanabe $\mathrm{S}$, Viet $\mathrm{H}$, et al. Human exposure to arsenic from drinking water in Vietnam. Sci Total Environ. 2014;488-489:562-9. doi 10.1016/j.scitotenv.2013.10.039.

12. Berg M, Luzi S, Trang T, Viet H, Giger W, Stuben D. Arsenic removal from groundwater by household sandfilters: comparative field study, model calculations, and health benefits. Environ Sci Technol. 2006;40(17):5567-73. doi: 10.1021/ es060144z.
13. Nitzsche S, Lan M, Trang K, Viet H, Berg $\mathrm{M}$, Voegelin A. et. al. Arsenic removal from drinking water by a household sand filter in Vietnam-Effect of filter usage practices on arsenic removal efficiency and microbiological water quality. Sci Total Environ. 2015;502:526-36. doi: 10.1016/j.scitotenv.2014.09.055.

14. Leupin X, Hug J. Oxidation and removal of arsenic (III) from aerated groundwater by filtration through sand and zero-valent iron. Water Res. 2005;39(9):1729-40. doi: 10.1016/j.watres.2005.02.012.

15. Leupin X, Hug J, Badruzzaman M. Arsenic removal from Bangladesh tube well water with filter columns containing zerovalent iron filings and sand. Environ Sci Technol. 2005;39(20):8032-7. doi: $10.1021 /$ es050205d.

16. Mehta $S$, Chaudhari K. Arsenic removal from simulated groundwater using household filter columns containing iron filings and sand. Journal of Water Process Engineering. 2015;6:151-7. doi: 10.1016/j jwpe.2015.04.008

Correspondencia: Guillermo Fernando Villa Gonzales.

Dirección: Calle Amapolas 350 Lince, Lima. Centro Nacional de Salud Ocupacional y

Protección del Ambiente para la Salud.

Teléfono: 748-0000, anexo 7722

Email:gvilla@ins.gob.pe 